\title{
Enhanced Förster resonance energy transfer between the CdTe quantum dots in proximity to gold nanoparticles
}

\author{
Vamsi K. Komarala ${ }^{1}$, A. Louise Bradley ${ }^{1}$, Yury P. Rakovich ${ }^{1}$, Stephen J. Byrne ${ }^{2}$, Yurii K. Gun'ko ${ }^{2}$ \\ and Andrey L. Rogach ${ }^{3}$ \\ ${ }^{1}$ Semiconductor Photonics Group, School of Physics, Trinity College Dublin, Ireland \\ ${ }^{2}$ School of Chemistry, Trinity College Dublin, Ireland \\ ${ }^{3}$ Photonics and Optoelectronics Group, Physics Department and CeNS, \\ Ludwig-Maximilians-Universitat, Munich, Germany
}

\begin{abstract}
Förster resonant energy transfer (FRET) between the CdTe quantum dot (QD) acting as donors and acceptors is investigated at nanoscale proximity to gold nanoparticles (Au NPs). Photoluminescence (PL) studies of the acceptor QD emission from a mixed monolayer showed a distance dependent enhancement of the acceptor emission compared with that achieved for a donor-acceptor mixed monolayer in the absence of the Au NP layer. Time-resolved photoluminescence measurements showing a reduction in the donor lifetime, accompanied by an increase in the acceptor PL lifetime, provide further evidence for surface plasmon enhanced FRET.
\end{abstract}

Key Words: Quantum dots, metal nanoparticles, surface plasmons, resonant energy transfer, exciton dipoles.

\section{INTRODUCTION}

There is growing interest in the use of semiconductor nanocrystals or colloidal quantum dots (QDs) in optoelectronic and bio-technology applications, such as light emitters ${ }^{1}$, lasers ${ }^{2}$, biological labeling and bio-sensing ${ }^{3}$. Förster resonant energy transfer (FRET) is an important communication and transport mechanism on the nanoscale. The control of this process in assemblies of QDs or on the single donor-acceptor pair level will play an important role in the development of many of these applications. FRET between QDs has been investigated in closely packed mixed layers and separated donor and acceptor layers ${ }^{4}$. Cascaded energy transfer in artificial solids formed from layers of colloidal QDs has been demonstrated ${ }^{5}$. Improvements in FRET have been concentrated on structures with reduced donor-acceptor separation, improved spectral overlap of the donor emission and acceptor absorption spectra and increased packing density of the layers. This has resulted in FRET times as fast as $\sim 50 \mathrm{ps}$ in close-packed 2D monolayers assembled using Langmuir-Blodgett techniques ${ }^{6}$ and 120 ps for QD bi-layers ${ }^{7}$.

We investigate the control of FRET between CdTe QD donors and acceptors using the influence of surface plamsons. Metal nanoparticles (NPs) of diameters much smaller than the optical wavelength of the excitation light support surface plasmons (SP) ${ }^{8}$. SP physics can be exploited to achieve enhanced Raman scattering, ${ }^{9}$ modified emission $^{10}$ and molecular labeling ${ }^{11}$ to name a few applications. There have been some theoretical studies of the potential to use SP supported by metal NPs to accelerate Förster/nonradiative resonance energy transfer (FRET) between the donor and acceptor emitters ${ }^{12}$.

Plasmonics: Metallic Nanostructures and Their Optical Properties V, edited by Mark I. Stockman, Proc. of SPIE Vol. 6641, 66410Y, (2007) · 0277-786X/07/\$18 - doi: 10.1117/12.732147 
We present evidence for enhanced FRET between the CdTe QDs at nanoscale proximity to Au NPs which support a surface plasmon resonance. The FRET efficiency between CdTe QD donors and acceptors in a mixed monolayer is compared for samples which incorporate a $\mathrm{Au}$ NP layer and for samples which are deposited directly on a quartz substrate. The structures studied are prepared using the layer-by-layer (LBL) electrostatic assembly technique. ${ }^{13} \mathrm{~A}$ polyelectrolyte spacer layer provides separation between the Au NP layer and the QD layers to quenching of the QD emission. FRET between the donor-acceptor pair is characterized using photoluminescence (PL) spectroscopy and timeresolved PL measurements.

\section{EXPERIMENTATION}

Water-soluble CdTe QDs were synthesized, using thioglycolic acid (TGA) as short-chain thiol stabilizers, which imports negative charge on the QD surfaces. These CdTe QDs have peak PL emission at $\sim 525 \mathrm{~nm}$ and $\sim 600 \mathrm{~nm}$ and acts as the donor and acceptor respectively. Further details of the growth conditions are reported elsewhere. ${ }^{14}$ Watersoluble Au NPs of size $\sim 7-8 \mathrm{~nm}$ exhibiting strong absorption at $\sim 520 \mathrm{~nm}$ and with a negative surface charge were also prepared by the reported procedure. ${ }^{15}$

We use a layer-by-layer (LBL) electrostatic self-assembly as a nanoscale fabrication technique ${ }^{13}$ for preparation of monolayers of Au NPs and CdTe. The adsorption of alternate positively and negatively charged polyelectrolyte (PE) layers provides nanoscale control of the thickness of the spacer layer separating the Au NP layer and the QD layer. Prior to the deposition of Au NPs layers, the quartz substrates are thoroughly cleaned/ultrasonicated with $\mathrm{NaOH}$ and methanol. This renders the substrate hydrophilic in nature and facilitates the growth of the monolayers. Three monolayers of $\mathrm{Au}$ NPs are deposited on the quartz substrate using polyethyleneimine (PEI) as a counterpart. The PEI solution is prepared by taking $1 \mathrm{ml}$ in $4 \mathrm{ml}$ water, for this $2 \mathrm{ml}$ of $3 \mathrm{M} \mathrm{NaCl}$ is added. Polyelectrolyte bi-layers are self-assembled to the required thickness by sequentially dipping a $\mathrm{Au}$ NPs coated quartz substrate for 20 minutes in Poly(diallyldimethylammonium chloride) (PDDA) and Poly(sodium 4-styrenesulfonate) (PSS) solutions. The PDDA solution is prepared by taking $150 \mu \mathrm{l}(20 \%$ weight in water) in $5 \mathrm{ml}$ water and the PSS solution is prepared with a concentration of $0.412 \mathrm{mg} / \mathrm{ml}$ in water. For each $5 \mathrm{ml}$ of PDDA and PSS solutions, $1 \mathrm{ml}$ of $3 \mathrm{M} \mathrm{NaCl}$ is added. By repeating these dipping procedures, multilayer films are assembled to the required thickness. The thickness is approximately $1.4 \mathrm{~nm}$ for a single PDDA/PSS bi-layer and $11.7 \mathrm{~nm}$ for a film composed of 9 PDDA/PSS bi-layers. ${ }^{13} \mathrm{In}$ this work we prepared films comprised of 4, 6, 8, 10, 12, 14, and 16 PE bi-layers. Since the donor and acceptor CdTe QDs are stabilized with TGA, resulting in a negative surface charge, the final PE layer of the multilayer structure is a positively charged PDDA layer. As a final step, a monolayer of mixed CdTe QDs, containing both the $525 \mathrm{~nm}$ emitting donors and the $600 \mathrm{~nm}$ emitting acceptors, is deposited on Au NPs/PE bi-layers structures. A reference sample, consisting of a monolayer of mixed CdTe QDs deposited on 2 PE bi-layers on a quartz substrate, is also prepared. In between each dip the sample rinsed with Millipore-water to remove any loose polyelectrolyte molecules, Au NPs and QDs not ionically bonded to the surface. The donor and acceptor concentrations in the mixed solution prior to deposition were selected so that the mixed monolayer would exhibit equal PL intensities for each species when deposited on the quartz substrates with no Au NP layer. The same QD concentrations were used for depositing the mixed QD monolayer on the samples with an Au NP layer and it is expected that identical mixed QD monolayers are formed in each case. Following the deposition of the mixed QD monolayer, the samples are vacuum dried for 10 minutes.

A Shimadzu spectrophotometer is used to record the absorption spectra. Room temperature photoluminescence (PL) spectra were recorded using a Perkin-Elmer fluorescence spectrometer. The excitation wavelength of $400 \mathrm{~nm}$ is provided by a Xenon lamp. The time resolved PL decays were measured using a PicoQuant Microtime200 time-resolved confocal microscope system, equipped with on Olympus IX71 inverted microscope. The samples were excited by $480 \mathrm{~nm}$ picosecond pulses, generated by a PicoQuant LDH-480 laser head which is controlled by a PDL- 800 B driver. The system has an overall time resolution of $\sim 150 \mathrm{ps}$. The typical scanning area was $80 \mathrm{X} 80 \mu \mathrm{m}$ with $150 \mathrm{X} 150$ lines. Lifetime maps are calculated on a pixel-by-pixel basis by fitting the lifetime of each pixel to the logarithm of the intensity. The PL decays are determined by nonlinear least-squares analysis using FluoFit, PicoQuant. The system time response is negligible compared with typical lifetimes of the QDs. Broad band filters at 500 and $600 \mathrm{~nm}$ are used in PL lifetime measurements to collect the emission from the donors and acceptors NCs respectively. 


\section{RESULTS \& DISCUSSION}

The absorption spectrum for three monolayers of Au NPs is shown in figure 1, along with the absorption and PL spectra for acceptor and donor QDs. The Au NPs SP absorption maximum is red shifted to $\sim 565 \mathrm{~nm}$ compared to the maximum at $\sim 520 \mathrm{~nm}$ observed for isolated Au NPs in solution. The red-shift results from near-field dipolar interactions between the neighboring particles when the spacing between the NPs is much less than the optical wavelength, as in a closely spaced monolayer. ${ }^{8}$ As can be seen in figure 1, the Au NPs SP absorption feature overlaps with the donor emission and acceptor QDs PL absorption spectra.

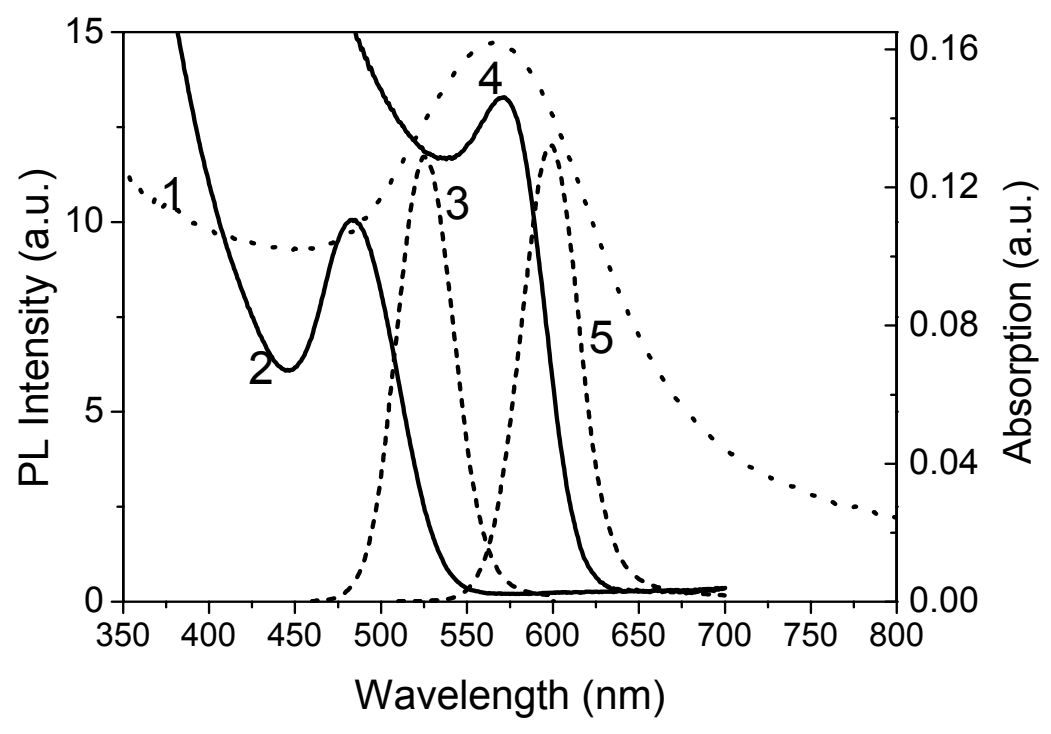

FIG. 1 The absorption spectrum for three monolayers of Au NPs exhibiting the surface plasmon resonance at 565 nm labeled as 1 (dots), donor CdTe NCs absorption (solid) and emission (dash) spectra in solution labeled as 2 and 3, acceptor CdTe NCs absorption (solid) and emission (dash) spectra in solution labeled as 4 and 5.

The PL spectra for the monolayer of mixed QDs on quartz is shown in Figure 2 shows. Two PL peaks are clearly visible corresponding to the emission for the donor QDs at $\sim 525 \mathrm{~nm}$ and the acceptor QDs at $\sim 600 \mathrm{~nm}$ respectively. The spectra were also recorded as a function of separation between the Au NP layer and the mixed QD layer. The spectra for 12,14 and 16 bi-layers spacer layers are shown. To compare energy transfer with and without the underlying Au NP layer the donor PL peak intensity for the samples including an Au NP layer is normalized to that of the sample with no $\mathrm{Au}$ NP layer. PL spectrum of acceptor QDs shows more than 4-fold enhancement in PL intensity at 12 PE bilayers $(\sim 20$ $\mathrm{nm}$ ) spacing from the Au NPs layer when compared to same mixed QDs on quartz substrate. Further increase in the thickness of the PE spacer layer causes a decrease in the acceptor PL intensity. For example for 16 PE bilayers the enhancement has dropped to a factor of 2. Therefore the enhancement factor is very sensitive to the distance between mixed QDs and Au NPs layer. The decrease in acceptor PL intensity after 12 PE layers spacing reflects the evanescent character of the SP dipole field from the Au NPs surface in z-direction. When the mixed QDs are less than $12 \mathrm{~nm}$ from the Au NPs layer, nonradiative energy transfer mechanism from QDs to Au NPs dominates and a quenching of the acceptor QD emission is observed. Samples with a pure monolayer of acceptor QDs have also been prepared and the PL as a function of separation from the Au NP layer has been investigated. In this case it was noted that even for a PE spacer layer thickness of 12 bi-layers there is still slight quenching of the acceptor PL emission and therefore the enhancement of the acceptor emission in the mixed QD monolayer cannot be attributed to the interaction between the acceptor QDs and the Au NP layer alone. 


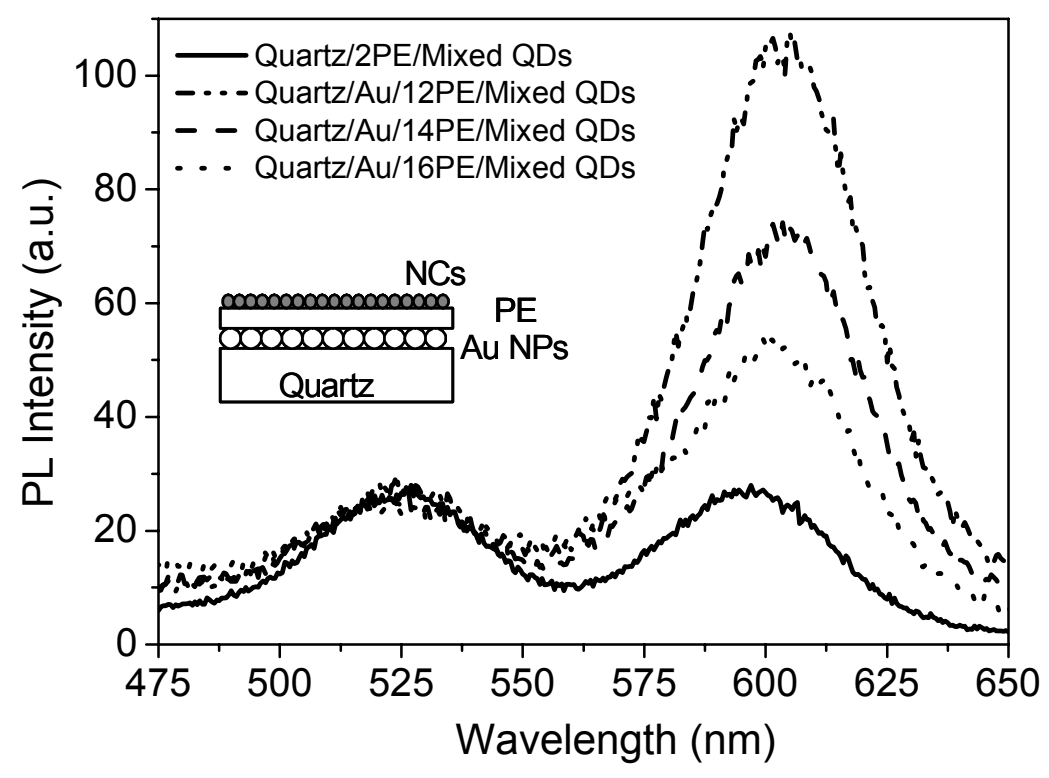

FIG. 2 Photoluminescence spectra for a mixed donor-acceptor CdTe NC layer in structures with and without the Au NP layer. The PL spectra with 12,14 and 16 PE bi-layers between the Au NP layer and the mixed QD layer are shown. A schematic of the sample structure is shown as an inset. The layer labeled PE corresponds to the PDDA/PSS polyelectrolyte bi-layers.

Figures 3 (a) and (b) show the PL decays for the donor and acceptor QDs in pure and mixed monolayers for samples with and without the Au NP layer. The measured average lifetimes for pure monolayers of donor and acceptor QDs in the absence of the Au NP layer are $5.74 \mathrm{~ns}$ and $4.87 \mathrm{~ns}$ respectively, in comparison with average lifetimes of $5.74 \mathrm{~ns}$ and $3.38 \mathrm{~ns}$ respectively for samples with an underlying Au NP layer. Therefore the inclusion of the Au NP layer has no effect on the donor PL lifetime and causes a reduction in the acceptor PL lifetime. No modification of isolated donor decay rate with the Au NPs layer indicates that the radiative and non-radiative process is not altered with Au NPs layer. In case of the mixed QD monolayer, the donor and acceptor lifetimes in the absence of the Au NP layer are $4.40 \mathrm{~ns}$ and $10.70 \mathrm{~ns}$ respectively, with PL lifetimes of $2.08 \mathrm{~ns}$ and $7.52 \mathrm{~ns}$ are obtained in the presence of the Au NP layer.

Figure 4 also shows the dependence of the donor average lifetime values on the separation between the mixed QD layer and $\mathrm{Au}$ NP layers in terms of the number of PE bi-layers. The average donor QD lifetime varies from $3.00 \mathrm{~ns}$ to a minimum of $2.00 \mathrm{~ns}$ for $\sim 10 \mathrm{PE}$ bi-layers. Also shown for comparison are the average lifetime values for a pure monolayer of donor QDs and for the donors QDs in the mixed QDs on quartz substrates with no Au NP layer. As can be seen the donor lifetime reduces from $5.74 \mathrm{~ns}$ for a pure monolayer of donor QDs to 4.40 ns for the mixed QD monolayer.

FRET causes a de-excitation of the donor and simultaneous excitation of the acceptor. As the Förster energy transfer rate is faster than the radiative rate this causes an increase in the donor decay rate and hence a reduced donor PL lifetime is a signature of FRET. ${ }^{17}$ A FRET efficiency, E, of $\sim 23 \%$ can be estimated using

$$
E=1-\frac{\tau_{D A}}{\tau_{D}}
$$


where $\tau_{\mathrm{DA}}$ and $\tau_{\mathrm{D}}$ are the donor lifetimes in the presence and absence of the acceptor. When the mixed QD monolayer is at $12 \mathrm{PE}$ bi-layers from the Au NP layer the donor lifetime is seen to decrease to $2.06 \mathrm{~ns}$ and the acceptor lifetime increases to $7.52 \mathrm{~ns}$. Taking the donor lifetime in the absence and presence of the acceptor in the Au NP layer structures, the FRET efficiency is estimated to be $\sim 64 \%$, representing almost a 3 fold enhancement, which is in reasonable agreement with the $\sim 4$ fold enhancement estimated from the PL spectral data. Clearly the Au NP layer has a strong influence on the donor-acceptor interaction. FRET from the donor to acceptor QDs also results in an increase in the acceptor lifetime, figure 3(b). In the absence of an Au monolayer the acceptor QD average PL lifetime is increased from $4.87 \mathrm{~ns}$ in a pure monolayer to $10.70 \mathrm{~ns}$ in the mixed donor-acceptor layer. In the presence of the Au NP layer the acceptor QDs average PL lifetime is increased from $3.38 \mathrm{~ns}$ in a monolayer to $7.52 \mathrm{~ns}$ in the mixed QD layer.
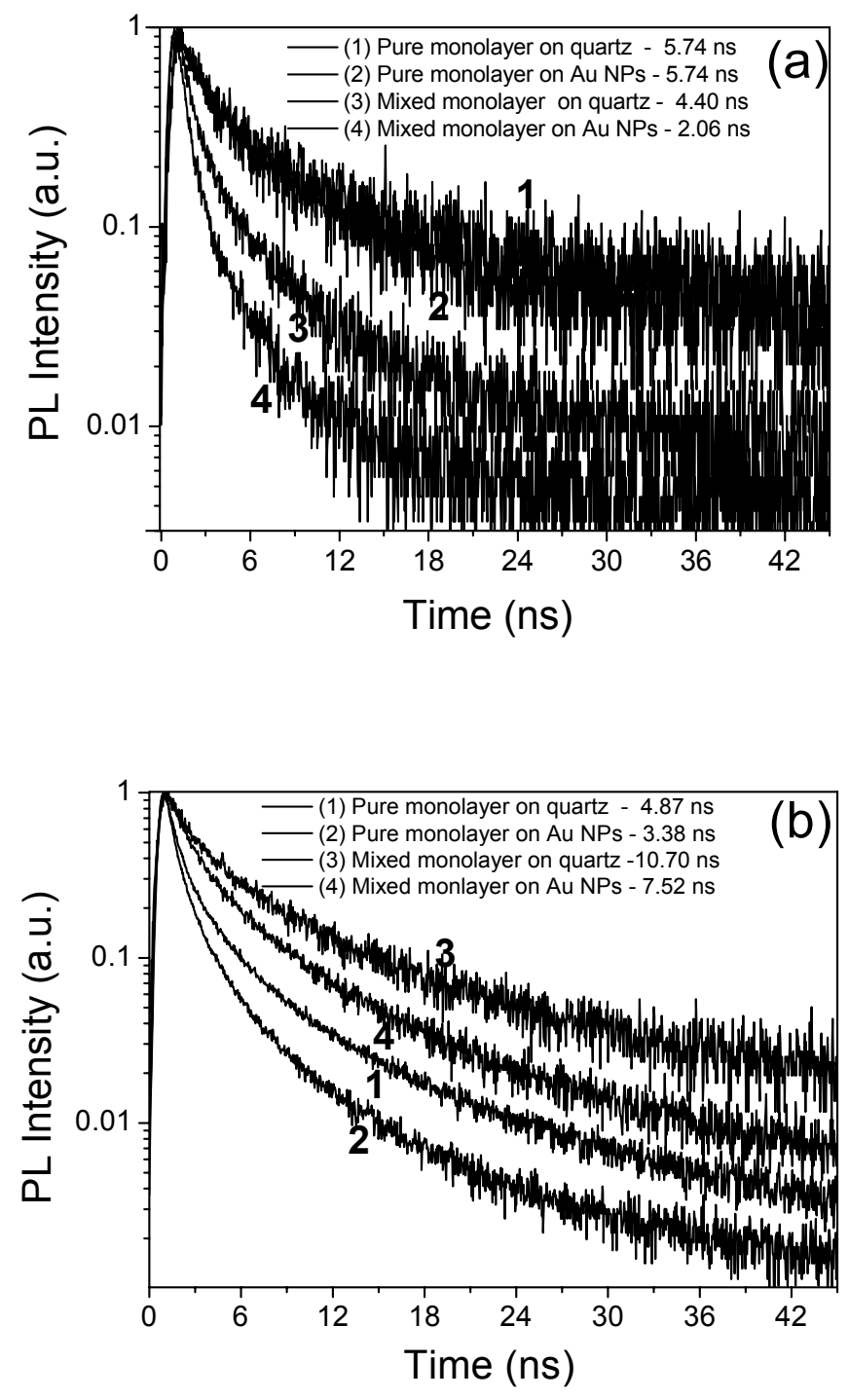

FIG. 3 Photoluminescence decays for donor CdTe NCs (a) and the acceptor CdTe NCs (b). The decays are labeled 1 to 4 and the average PL lifetime for each decay is given in the legend. 1: pure donor or acceptor NC monolayer with no Au NP film underneath, 2: pure donor NC or acceptor NC monolayer on Au NP film, 3: mixed NC monolayer with no Au NP film underneath, 4: mixed donor-acceptor NC monolayer on Au NP film. The separation between the Au NP film and the NC monolayer is $20 \mathrm{~nm}$ where relevant. 
The mechanism for non-radiative energy transfer fundamentally arises from the donor and acceptor transition moments. ${ }^{17}$ This process is mediated by the near-field related to the excited QD dipoles. The energy transfer rate via dipole-dipole interaction can be expressed as

$$
k_{\text {Förster }}=\frac{2 \pi}{\hbar} \frac{\mu_{D}^{2} \mu_{A}^{2} \kappa^{2}}{r^{6} n^{4}} \phi
$$

where the $\mu_{\mathrm{D}}$ and $\mu_{\mathrm{A}}$ are dipole moments of donors and acceptors, $\kappa^{2}$ is the dipoles orientation factor, $\phi$ is the spectral overlap integral (the donor emission should overlap with acceptor absorption spectrum for efficient excitation transfer), $\mathrm{n}$ is the refractive index of the medium and ' $\mathrm{r}$ ' is the distance between the donor and acceptor dipoles. ${ }^{17}$ Under our experimental conditions, we can consider that while there is a distribution of donor-acceptor distances of a mixed QD layer, this distribution is unchanged by the incorporation of an Au NP layer. we assume that the distribution of donor-acceptor separations within the mixed QD monolayer is unchanged by the incorporation of an Au NP layer. Likewise it is also expected that the orientation of the transition dipoles and refractive index of the emitting layer remain unchanged.

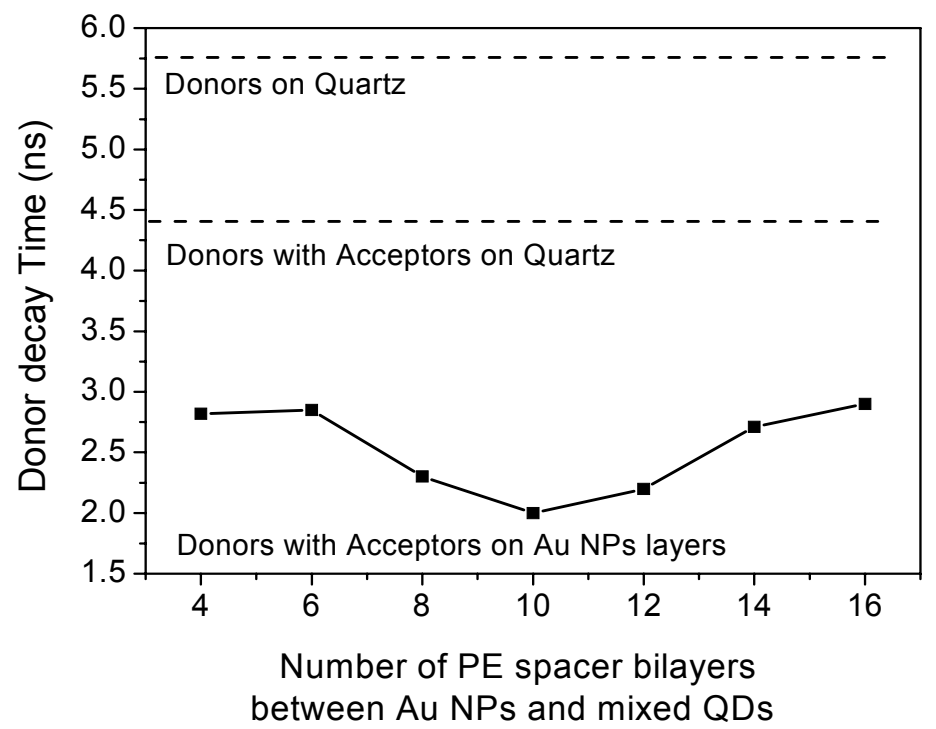

Figure 4: Average donor lifetimes for pure QD monolayer and for a mixed QD monolayer in samples with no Au NP layer are shown by the dashed lines. The average donor lifetime in a mixed QD monolayer as a function of PE bilayers in samples with an Au NO layer are also shown (匹).

The increased FRET efficiency is attributed to increased coupling of the donor and acceptor dipoles through the induced SP dipole field supported by the Au NPs. In our case the SP dipole frequency has strong overlap with both the donor (emitting) and acceptor (absorbing) transition frequencies as shown in Fig.1. These SP dipole fields can be very intense and larger than the QD exciton dipole fields, especially in extended systems such as layers with nanoscale roughness as in this case. The near-fields produced around Au NPs seem to drive and couple the donor-acceptor electronic transition states efficiently.

The spin-orbit splitting, phonon confinement and surface states all vary with the size of the QDs. ${ }^{18}$ The large inhomogeneous broadening of the QD spectra limits on the FRET efficiency in nanocrystal solids as even though the 
ensemble emission and absorption spectra overlap, only a small fraction of the individual donor and acceptor spectra have a significant overlap integral. In mixed QD layers, such as those studied in this letter, the FRET efficiency is further reduced as the resonant acceptors may not be sufficiently close to the donors. The SP interaction with the donoracceptor pair provides a mechanism to compensate for these factors.

\section{CONCLUSIONS}

In conclusion we have investigated FRET between semiconductor colloidal CdTe QDs in proximity to Au NPs using steady state and time-resolved PL measurements. The FRET efficiency is strongly dependent on the separation between the monolayer of mixed donor and acceptor QDs and the Au NP layer. The maximum FRET efficiency occurs for a separation of $\sim 20 \mathrm{~nm}$ and decreases rapidly for larger separations. From the PL spectral data a maximum $\sim 4$ fold enhancement in the FRET efficiency between the donor and acceptor QDs is observed with a $20 \mathrm{~nm}$ separation. This has been further supported by the time-resolved PL data with the donor QD average lifetime reduced to its minimum value at this Au NP layer-QD layer separation. The characteristic spectra overlap with the SP absorption of the Au NP layer and the modified or enhanced dipole (emitting)-dipole (absorbing) interaction between the donor and the acceptor QDs is attributed to the presence of strong SP dipole fields. The possibility to enhance the FRET process through SP interactions can lead to improvements in many QD based optoelectronic and biological applications. For example, artificial solids for energy funneling and light harvesting devices can be designed to exploit this effect.

\section{Acknowledgments:}

This research is funded by Science Foundation of Ireland (SFI) under grant SFI 05/PICA/1797. A.L. Rogach acknowledges the SFI Walton Award. We thank Andrei Susha for providing gold nanoparticles for this study.

\section{REFERENCES}

1. V. L. Colvin, M. C. Schlamp, A. P. Alivisatos, Nature 370, 354 (1994), S. Coe, W.K. Woo, M. Bawendi and V. Bulovic, Nature 420, 800 (2002).

2. A.V. Malho et al., Appl. Phys. Lett. 81, 1303 (2002), V. I. Klimov et al., Science 290, 314 (2000).

3. I. L. Medintz et al., Nature Mat. 2, 630 (2003); A. P. Alivisatos, Nature Biotechnology 22, 47 (2004).

4. C. R. Kagan, C. B. Murray, M. Nirmal and M. G. Bawendi, Phys. Rev. Lett. 76, 1517 (1996); S. A. Crooker, J. A. Hollingsworh, S. Tretiak and V. I. Klimov, Phys. Rev. Lett. 89, 86802 (2002); T. Franzl, D. S. Koktysh, T. A. Klar, A. L. Rogach and J. Feldmann, Appl. Phys. Lett. 84, 2904 (2004).

5. T. Franzl , S. Schietinger, A. L. Rogach and J. Feldmann, Nano Lett. 4, 1599 (2004).

6. M. Achermann, M. A. Petruska, S. A. Crooker and V.I. Klimov, J. Phys. Chem. B 107 13782-13787 (2003);

7. T. Franzl, A. Shavel, A. L. Rogach, N. Gaponik, T. A. Klar, A. Eychmüller and J. Feldmann, Small 1, 392 (2005).

8. U. Kreibig and M. Vollmer, Optical properties of metal clusters; Springer-Verlag: Berlin, 1995.

9. S. Nie and S. R. Emory, Science 275, 1102 (1997).

10. V. K. Komarala, Y. P. Rakovich, A. L. Bradley, S. J. Byrne, Y. K. Gun'ko, N. Gaponik and A. Eychmüller, Appl. Phys. Lett. 89, 253118 (2006). 
11. G. L. Liu et al., Nature Nanotech. 1, 47 (2006)

12. J. I. Gersten and A. Nitzan, Chem. Phys. Lett. 104, 31 (1984); H. M. Hua, J. I. Gersten and A. Nitzan, J. Chem. Phys. 83, 3650 (1985).

13. G. Decher, Science 277, 1232 (1997).

14. S. J. Byrne, S.A. Corr, T.Y. Rakovich, Y.K. Gun'ko, Y.P. Rakovich, J.F. Donegan, S. Mitchell and Y. Volkov, J. Mater. Chem., 16, 2896 (2006); N. Gaponik, D. V. Talapin, A. L. Rogach, K. Hoppe, E. V. Shevchenko, A. Kornowski, A. Eychmuller and H. J. Weller, J. Phys. Chem. B 106, 7177 (2002).

15. D. I. Gittins, and F. Caruso, Angew. Chem. Int. Ed. 40, 3001 (2001).

16. F. Caruso, Adv. Mater. 13, 11 (2001).

17. Th. Förster, Ann. Phys. 2, 55 (1948); D. L. Andrews, A. A. Demidov, Resonance Energy Transfer; Wiley: Chichester, U. K., 1999.

18. Al. L. Efros, M. Rosen, M. Kuno, M. Nirmal, D. J. Norris and M. Bawendi, Phys. Rev. B 54, 4843 (1996); T. Takagahara Phys. Rev. Lett. 71, 3577 (1993), M. G. Bawendi, P. J. Caroroll, W. L. Wilson and L. E. Brus, J. Chem. Phys. 96, 946 (1992). 